\title{
Critical review of multidisciplinary non-surgical local interventional ablation techniques in primary or secondary liver malignancies
}

\author{
Attila Kovács, MD!, Roberto lezzi, MD23, Francesco Cellini, MD, Valentina Lancellotta, MD4, Peter Bischoff, MD!, \\ Francesca Carchesio, MD², Luca Tagliaferri, MD4, György Kovács, MD55, Maria Antonietta Gambacorta, MD 4,6 \\ IKlinik für Diagnostische und Interventionelle Radiologie und Neuroradiologie, Mediclin Robert Janker Klinik, Bonn, Germany, ${ }^{2}$ Fondazione \\ Policlinico Universitario A. Gemelli IRCCS, UOC di Radiologia, Dipartimento di Diagnostica per Immagini, Radioterapia Oncologica ed \\ Ematologia, Roma, Italia, ${ }^{3}$ Università Cattolica del Sacro Cuore, Istituto di Radiologia, Roma, Italia, ${ }^{4}$ UOC Radioterapia Oncologica, \\ Dipartimento di Diagnostica per Immagini, Radioterapia Oncologica ed Ematologia, Fondazione Policlinico Universitario A. Gemelli IRCCS, \\ Rome, Italy, ${ }^{5}$ Gemelli INTERACTS - Università Cattolica del Sacro Cuore, Rome, Italy, ${ }^{6}$ Università Cattolica del Sacro Cuore, Rome Italy
}

\begin{abstract}
Local non-surgical interventional percutaneous ablation represents nowadays an important part of the potential treatment strategies. Although surgical ablation represents the gold standard, in the past decade there was an expansion in the use of non-surgical ablative techniques: radiofrequency, microwave, laser, cryoablation, irreversible electroporation, and interventional radiotherapy (brachytherapy) in primary as well as secondary liver cancers. With the growing experience in the field, there was implemented a new pillar for cancer treatment, together with surgery, chemotherapy as well as radiotherapy, so-called interventional oncology (IO). To date, there are no published papers regarding a comparative interdisciplinary evaluation of all these non-surgical interventional local ablation therapies. Our paper offers a critical interdisciplinary overview of the treatments in both primary and secondary liver tumors, including from a cost-effective point of view. Furthermore, the present status of education in IO and a comparison of actual economic aspects of the treatments are also provided.

J Contemp Brachytherapy 2019; 11, 6: 589-600 DOI: https://doi.org/10.5114/jcb.2019.90466
\end{abstract}

Key words: liver percutaneous ablation, liver brachytherapy, interventional oncology treatment costs, education.

\section{Purpose}

Although surgical resection represents the gold standard, in the past decade there was an expansion in the use of non-surgical ablative techniques in primary as well as secondary liver cancers. With the growing experience in the field, the importance of multidisciplinary teams of specialized centers was realized in an optimal treatment procedure, implementing the so-called interventional oncology (IO).

Due to the close cooperation between interventional radiology and interventional radiotherapy (brachytherapy) experts, beside widely accessible 3D-conformal or stereotactic external beam radiation $[1,2,3]$, local non-surgical interventional tumor tissue ablation represents nowadays an additional option for the treatment of patients with cancer, together with surgery, chemotherapy, and radiotherapy. Few years ago, percutaneous interventional ablation techniques were exclusively, indicated as palliative and experimental treatments [4]. However, today the scenario has completed changed, with ablation treat- ments also used as a first-line option in selected tumors and selected patients $[5,6]$. These treatments are also suitable for nonsurgical well-selected patients, with technical or clinical restrictions, based on multidisciplinary tumor board decision $[7,8,9,10]$. Furthermore, in some cases IO treatments are also preferred from a cost-effective point of view [11]. Radiation therapy is preferably performed in most of the institutions as external beam stereotactic radiotherapy [12], and high levels of evidence do exist in different primary tumor anatomic sites and stating the survival advantage of treating oligometastatic disease, if the primary is stable $[13,14]$. However, interstitial computed tomography $(\mathrm{CT})$ guided implantation and irradiation has been described as an appropriate and successful method of local radiation [15]. However, percutaneous liver stereotactic body radiotherapy (SBRT) is a successful method in treating liver targets [16]. It is assumed that a rapidly increasing future workload of external beam facilities will allow an optimal patient service, and spare valuable external beam facility time [17]. 
Additionally, the use of brachytherapy can reduce the number of treatments needed, resulting in fewer visits to the radiotherapy department, potentially increasing the number of patients recruited [18]. Also the need for dose escalation as a biologically equivalent dose (BED) of 100 and possibly higher in some histologies is needed for control, which can easily be achieved with brachytherapy in the case of multiple targets or following previous radiation treatments [19].

There is a lack of comparative interdisciplinary evaluation of all these non-surgical interventional local ablation methods. The present paper would like to offer a critical interdisciplinary overview of the treatments in both primary as well as secondary liver tumors, including from a cost-effectiveness point of view.

\section{Percutaneous ablation treatments}

Ablation technology has rapidly evolved during the past few years, with substantial technical and procedural improvements that can help to increase clinical outcomes and safety profiles. The future of ablation should be based on an attempt to expand clinical indications as appropriate, creating the potential for larger areas of ablation, attempting to more precisely control the area of ablation, ensuring the safety of the procedure, and achieving even greater long-term success in terms of complete response rate, progression-free survival, and overall survival [20]. Recent developments include several new techniques to improve treatment efficacy. Within the classification of tumor ablation, several modalities are used worldwide: radiofrequency, microwave, laser, cryoablation, and irreversible electroporation. In detail, thermal energy is used to heat (radiofrequency, microwave, laser) or cool (cryoablation) tissue to cytotoxic levels (more than $60^{\circ} \mathrm{C}$ or less than $-40^{\circ} \mathrm{C}$ ). Alternative non-thermal technique is represented by irreversible electroporation, which targets the permeability of the cell membrane. Most ablation systems are based on a generator and a needlelike device that delivers the energy directly to the targeted tissue to achieve acute cellular necrosis. Each technique, although similar in purpose, has specific and optimal indications, with different advantages and disadvantages, which need to be briefly discussed.

\section{Radiofrequency ablation}

Radiofrequency ablation (RFA) is considered the firstline ablation technique, with advantages and limitations well demonstrated in experimental animal studies and clinical trials. It is based on low frequency radio waves with a long wavelength to determine heat, by ionic agitation with the adjacent molecules closer to the applicator, resulting in frictional indirect heating, with consequent coagulative necrosis and cell death [21,22,23]. RFA is characterized by a limited necrotic volume provided, eventually requiring multiple probes for lesions larger than $3 \mathrm{~cm}$ in size, with limitations mainly related to the heat sink effect as well as the risk for tumor carbonization, based on excessive desiccation of tissues which increases impedance and decreases the electrical current flow. Specific advantages are represented by well-proved safety and reproducibility/procedural standardization, with optimal results for primary and secondary hepatic tumors up to $3 \mathrm{~cm}$ in size; it is also characterized by a simple technique, low-profile/minimal-invasive ablative probe, as well as low cost of a single needle. The multiple probe approach can be used in order to treat larger lesions [24,25,26,27,28,29]. Major complications of RFA include intraperitoneal bleeding, infections, liver failure, pneumothorax, organ injury, bile duct stenosis and tumor lysis syndrome; however, major complication and procedural mortality rates are significantly low $[30,31]$.

\section{Microwave ablation}

Microwave ablation (MWA) is becoming a competitor to RFA; its mechanism of action is based on rotation or oscillation of water particles, resulting in direct heating. It is not limited by carbonization, with consequent faster heating and higher temperature available, with better propagation of electromagnetic waves. It is less influenced by tissue properties and not influenced by the heat sink effect. Finally, the increased thermal conductivity combined with increased energy deposition potentially create larger coagulation necrosis with potential better clinical results in terms of the local effect obtained. Despite the theoretical advantages from these improvements in MWA, to date, it remains unclear whether these are associated with actual clinical benefits in outcome. Randomized trials and meta-analyses showed equivalent therapeutic effects and complication rates between MWA and RFA ablation in the treatment of hepatocellular carcinoma (HCC) and colorectal liver metastases respectively $[32,33,34,35,36,37]$. This lack of agreement may be related to changes in generators/antennas used in the various studies. However, some interesting potentially beneficial features of MWA in comparison with RFA can be considered, including the shorter procedural duration and the easy placement of one straight antenna to obtain volumes of ablation that would require more than one RFA cooled electrode or expandable electrodes.

To date, MWA seems to be preferred for HCC larger than $3 \mathrm{~cm}$ in size or for metastatic lesions up to $4 \mathrm{~cm}$ in size (Figure 1). It needs to be underlined that for HCC larger than $3 \mathrm{~cm}$ in size it could also be used as combined treatment, performing in a single-step approach both chemoembolization and ablation, with a low complication rate and high CR rate obtained [38,39], also available in patients with complex lesions or with high risk of procedural bleeding [40].

\section{Cryoablation}

Cryoablation is the only technique which uses freezing instead of heat to create tumor necrosis. Its mechanism of action is based on the Joule-Thompson effect with the use of a gas, such as argon, which rapidly decreases to subzero temperatures (as low as $-150^{\circ} \mathrm{C}$ ) upon transition from a liquid to gaseous state; the freezing is alternated with a thaw cycle, during which helium gas is administered to raise the temperature to about $40^{\circ} \mathrm{C}$. 

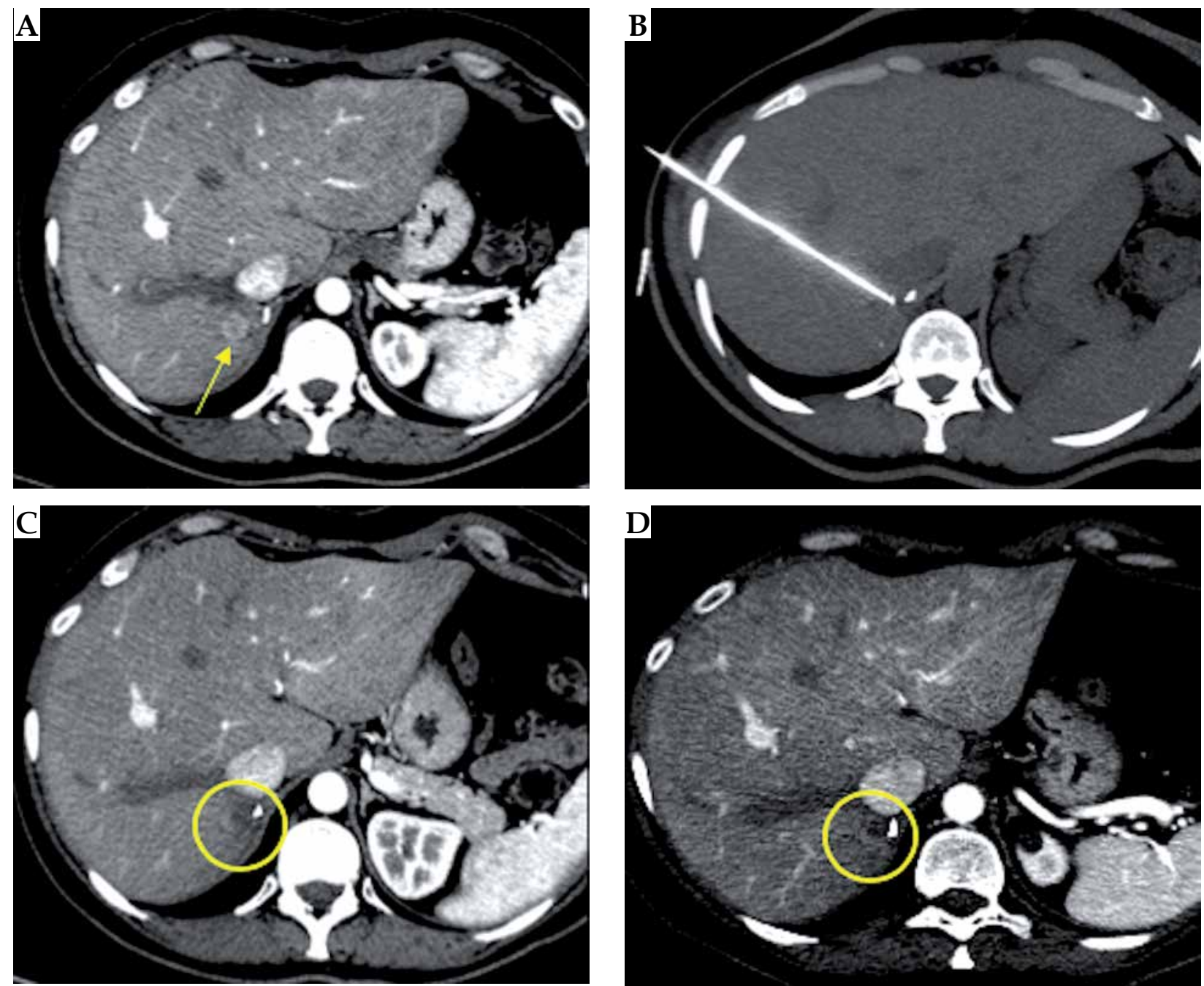

Fig. 1. Single liver metastasis from adrenal cancer in the VIIIs, close to inferior cava vein in a 43-year-old man. Contrast enhanced CT image in axial plane (A) shows hypervascular focal lesion $2 \mathrm{~cm}$ in size (arrow). The patient underwent percutaneous MWA ablation of the lesion (B). Contrast-enhanced CT examinations performed 3 months (C) and 12 months (D) after the procedure demonstrate complete necrosis without residual enhancing tissue in the ablated area, also preserving patency of inferior cava vein (post-treatment complete response)

The treatment protocol is composed of two 10-minutes cycles of freezing with a 5-minute break of thawing. Cell death is obtained with both direct and indirect mechanisms. In detail, rapid freezing determines the formation of intracellular/extracellular ice crystals with consequent disruption of the cell membrane and internal processes. Furthermore, indirect mechanisms include vasoconstriction and occlusion of blood vessels resulting from osmotic changes and local tissue edema resulting in hypoxic tissue injury and coagulative necrosis. Finally, they also generate immunological interactions and promotion of inflammatory cytokines, which may also exert a tumoricidal effect $[41,42,43]$.

The main advantages of the procedure are represented by: a) the much shorter intraprocedural pain, requiring only mild sedation, in particular for lesions near the diaphragm or abdominal wall, b) the tissue preservation and healing with resistance of collagenous architecture, c) the direct intraprocedural visualization of "core necrot- ic volume" on CT or MRI without contrast medium, with better necrosis predictability and control. Disadvantages are represented by the limited necrotic volume obtained (maximum transverse diameter obtained: less than $2 \mathrm{~cm}$ in size), with the need to use a multiple probe approach, the bulkiness of equipment (gas), the longer time of the procedure (more than 30 minutes versus less than 12 minutes for RFA or MWA), and the higher cost of the device (more expensive than RFA and MWA), also considering the need of multiple probes for obtaining a volume necrosis available for the treatment of a nodule of $3-4 \mathrm{~cm}$ in size.

Some authors have postulated that there may be a reluctance to treat hepatic tumors with cryoablation, perhaps because of concerns for bleeding, liver fracture, and cryoshock that were observed many years ago during open cryosurgery [44]. However, these severe complications typically occurred after cryoablation of large tumors and involved the placement of applicators as large 
as $9 \mathrm{~mm}$ in diameter, much larger than those currently percutaneously used [45]. A single prospective randomized clinical trial compared percutaneous cryoablation to RFA for the treatment of HCC, obtaining a similar overall 5-year survival rate [46].

Based on the intraprocedural monitoring of ice ball formation minimizing the chance of harming adjacent critical structures, the limited ice ball extension beyond the liver into the diaphragm or body wall with less pain and injury than RFA [47], cryoablation could be preferred to RFA or MWA to treat hepatic masses close to critical structures, such as the diaphragm, chest wall, heart, lung, gallbladder, and biliary tree $[48,49]$, with better results for lesions less than $4 \mathrm{~cm}$ in size [50] (Figure 2).

\section{Laser interstitial thermal therapy (LITT)}

With laser ablation, direct heating destroys tissue with low-power and highintensity laser light energy delivered percutaneously via thin optical fibers, with a diameter from 300 to $600 \mu \mathrm{m}$. Conventional bare-tip fibers provide an almost spherical thermal lesion of $12-15 \mathrm{~mm}$ in diameter, and a beam-splitting device or a multi-source device allow for the use of up to four fibers, simultaneously delivering the light into each single fiber.

Laser thermal ablation (LTA) technique, as proposed by Pacella et al. [51] and improved by Di Costanzo et al. [52], consists of $300 \mu \mathrm{m}$ bare optical fibers introduced into the tumor through 21-gauge needles. The diameter of the needles is smaller than RFA electrodes and MWA antennas, making LTA safer and more suitable for ablating lesions in at-risk locations or in locations that are difficult to reach [53]. Moreover, a multisource device allows from one to four fibers to be used at once, making it possible to achieve ablation areas from one to $4-5 \mathrm{~cm}$ in diameter, and consequently to treat tumors ranging from $5-6 \mathrm{~mm}$ to $3 \mathrm{~cm}$ in diameter, obtaining an acceptable safety margin.
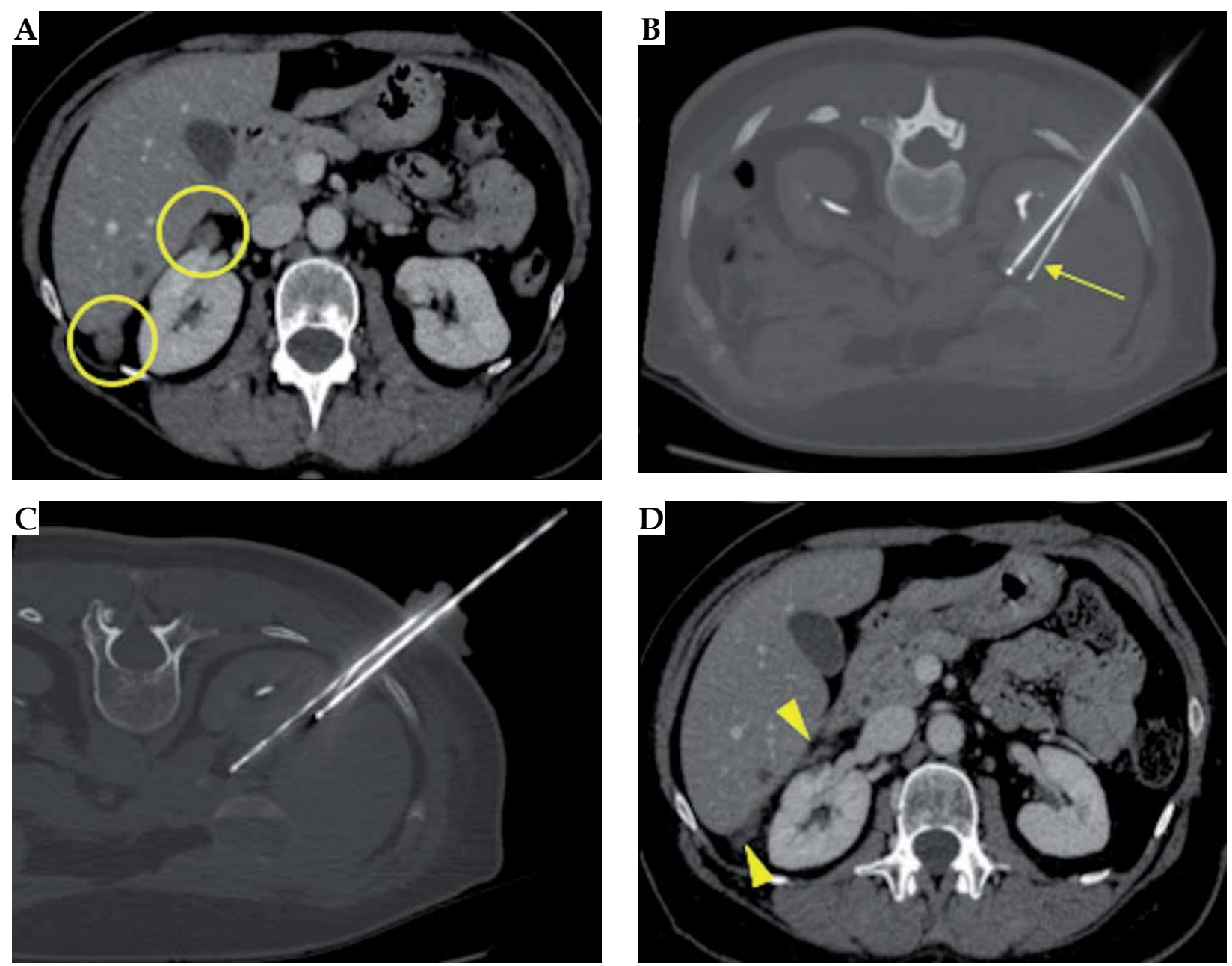

Fig. 2. Ovarian metastatic implants in the perihepatic visceral peritoneum mimicking hepatic metastases in a 58-year-old woman. Axial post-contrast CT image (A) shows two subserosal hepatic deposits of ovarian cancer with rounded, well-defined margins, $12 \mathrm{~mm}$ in size (circles). In order to obtain displacement of neighboring right kidney and duodenum, preventing untargeted ablation, a percutaneous hydrodissection using a Chiba needle (arrow) was performed (B). Thus, a simultaneous ablation of the two lesions was performed using two different cryoprobes (IceSphere, 2 freezing/thawing cycles) (C) obtaining, on 6-month follow-up contrast-enhanced CT examination (D), a complete response, without signs of residual viable tumor (arrowhead) 
Laser thermal ablation could be proposed as a valid alternative to RFA for lesions up to $2 \mathrm{~cm}$ [54], and it has been suggested as the technique of choice in the presence of multiple small and variably sized liver tumors. It needs to be underlined that the efficacy of LTA can be limited by the heat-sink effect, like RFA.

\section{Electroporation}

Reversible (RE) or irreversible (IRE) electroporation is a non-chemical non-thermal image-guided ablation technique [55]. Reversible electroporation is characterized by the creation of temporary and limited pathways for molecular transport via nanopores, but after the end of the electric pulse, the transport ceases and the cells remain viable. Medical applications include, for example, local introduction of intracellular cytotoxic pharmaceuticals such as bleomycin (electroporation and electrochemotherapy).

On the other hand, IRE is characterized by a certain degree of damage to the cell membranes by electroporation.

The leakage of intracellular contents could be too severe or the resealing of the cellular membrane too slow, leaving healthy and/or cancerous cells irreversibly damaged. They die by apoptosis, which is unique to this ablation technique, in opposition to all other ablation systems, which induce necrosis either by heat, cooling, or radiation.

It is a method to induce irreversible/reversible disruption of cell membrane integrity by changing the transmembrane potential resulting in cell death without the need for additional pharmacological injury. Because IRE is potentially a non-thermal technique, issues associated with perfusion-mediated tissue cooling or heating (a significant challenge with thermal methods) are less relevant. So far, the published data of the technique are mostly represented by case series including tumors in difficult perivascular or peribiliary locations [56,57].

Devices for IRE are more expensive than other ablative needles.

\section{Electrochemotherapy}

Electrochemotherapy (ECT) is also a non-thermal ablation, similar to a chemoablation, as a chemotherapeutic agent accomplishes the anticarcinogenic effect. ECT is a locally enhanced chemotherapy that combines the administration of a chemotherapeutic drug with cell membrane electroporation (MEP). MEP is the exposure of biological membranes to high external electric pulses, resulting in a destabilized surface tension and an increase in permeability of the cell membrane [58]. The latter is used to transfer into the cytosol large hydrophilic molecules such as bleomycin (BLM) or cisplatin (CIS) that are usually too big to cross the intact cell membrane. Regarding the technical implementation, after intravenous or intratumoral administration of the dedicated anticancer drug electrodes located around or inside the tumor deliver defined electric pulses which enables the cell membrane permeation and consequently the diffusion of otherwise non-permeant drugs into the target cells. Although in ECT the increase in cell membrane permeability is only temporary and reversible, the cytostatic drug once located intracellularly will cause multiple DNA breaks (BLM) or intra- and inter-strand DNA bonds (CIS). MEP in superficial lesions is achieved by the use of electrodes with a fixed geometry, while in deep-seated lesions it is achieved by interaction of single electrodes. Tumor size, location and histological type as well as electrode geometry, number, and spacing determine the effectiveness of ECT. Especially in deep-seated targets parallel long-needle positioning needs the skills and the support of image guidance. The applied electric field needs to be precisely planned to ensure complete and homogeneous coverage of the tumor volume. Dedicated software calculates the optimized placement of the electrodes as well as the voltage for each couple of electrodes. ECT allows controlled locoregional chemotherapy without marked systemic side effects. Moreover, one major benefit of ECT is a low dose of cytostatic drug infused due to a locally increased cytotoxic effect (in vitro 700-fold increase of toxicity in BLM and up to 12-fold in CIS), as only a few molecules per cell are needed to induce cell death [59].

Electrochemotherapy is already well established for cutaneous and subcutaneous primaries and metastases [60]. Preliminary results of the limited publications show that ECT in the liver is also feasible and safe. Data suggest effective local tumor control without compromising the quality of life of patients [61]. In patients with HCC (open surgery) ECT combined with BLM obtained a complete response of $88.2 \%$ per lesion [62], in patients with unresectable colorectal liver metastases (CRLM) of 85\% [63]. The analysis also revealed that after ECT treatment, most vessels $(>5 \mathrm{~mm})$ and biliary structures were preserved [64]. These findings suggest that ECT can be indicated for the therapy of metastases near major blood vessels in the liver to provide a safe approach with good antitumor efficacy (Figure 3)

Electrochemotherapy requires general anesthesia due to the electric pulses necessary for MEP. Further, the pulses may interfere with the heart-internal excitation-conduction-system; for that reason ECG-synchronization is mandatory to ensure the pulse delivery in the refractory phase of the heart. BLM may cause lung fibrosis; a higher BLM dosage, older age, and renal insufficiency seem to engrave the risk. BLM has a maximum lifetime dose of $350 \mathrm{mg}$. IRT)

Interstitial interventional radiotherapy (brachytherapy,

Interstitial interventional radiotherapy (IRT) is also a nonthermal ablation, rather a radioablation. It is an interventional image-guided internal radiation technique for local radiation treatment using high-dose-rate remote afterloading technology. The IRT radiation sources (usually $370 \mathrm{GBq}$ initial activity ${ }^{192} \mathrm{Ir}$ ) are temporarily placed via inactive applicators directly into the tissue of concern. The inherent property of IRT is to deliver a very high dose around the source positions and due to the rapid dose fall-off to spare sensitive normal tissues [65]. 

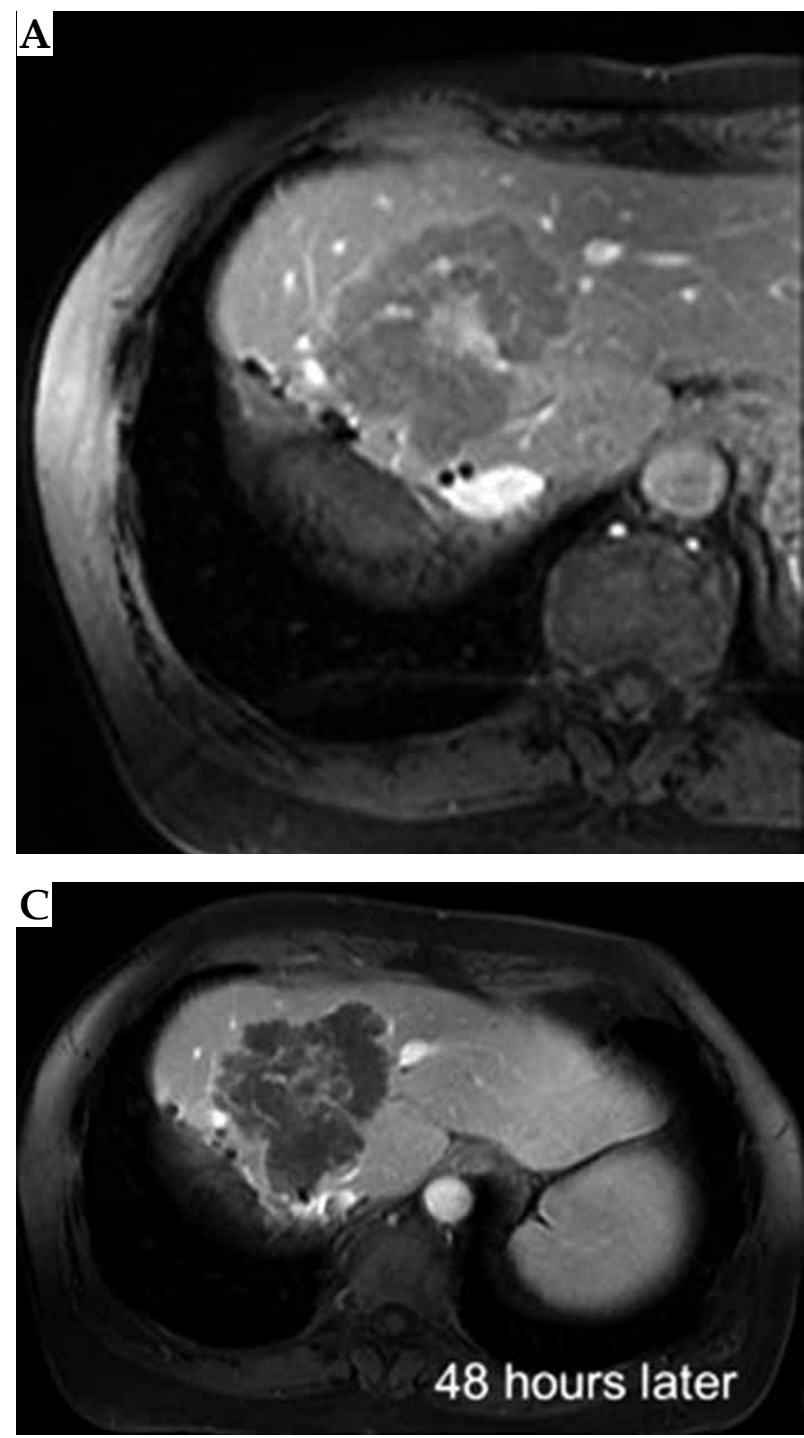

The optimal placement of the inactive IRT catheters should follow a certain geometry to offer ideal target volume distribution of the potential source positions within the target volume and is usually carried out in the CT scan room under aseptic precautions and under sedation. Once the implantation of the target is completed a thin-slice CT scan is done for dose distribution planning in an adequate treatment planning software package (TPS). The delineation of the clinical target volume (CTV) comprising the gross tumor volume (GTV) and the organs at risk (OARs) is done. After the catheter and anatomy reconstruction in the TPS, a volume optimized dose distribution is created. All modern remote HDR afterloading machines are eligible to deliver the radiation. Typical fraction doses covering the target are 15-20 Gy and usually a single fraction treatment is applied. Due to the steep dose fall-off of the used radioactive source, $>50$ Gy in the central tumor region results in the clearance of the central (hypoxic and less radiosensitive) parts of the tumor [66]. The duration of the radiation ranges from 20 to 60 minutes depending on the size of the CTV and the actual activity of the iridium source. The tumor

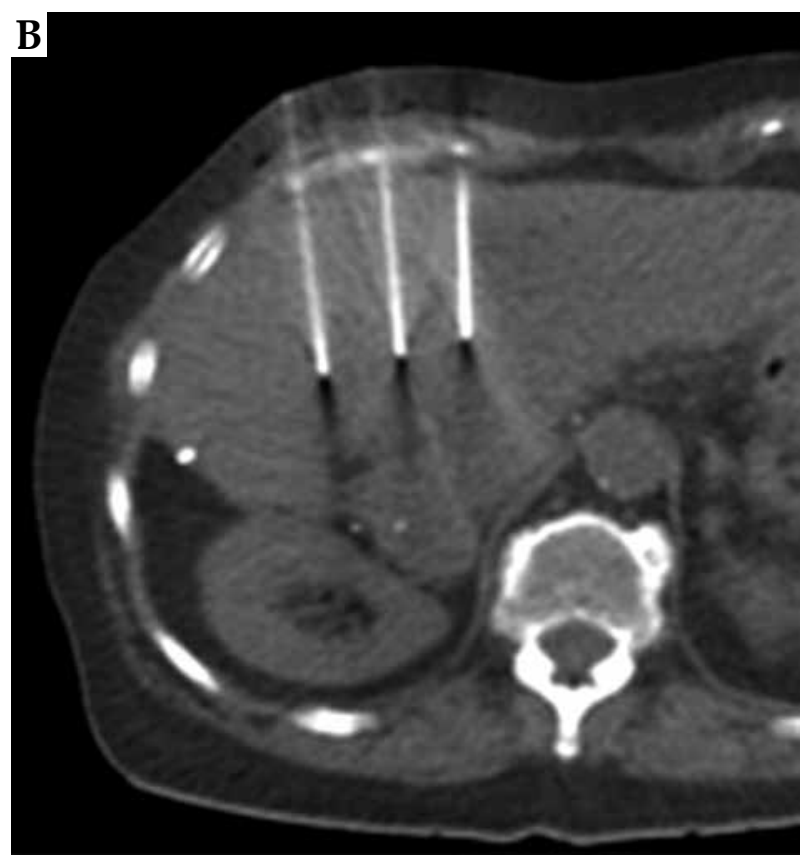

Fig. 3. A) Recurrent colorectal liver metastasis at the resection margin after hemihepatectomy. Systemic chemotherapy was exhausted and not well tolerated. B) After intravenous administration of bleomycin electric pulses were deployed through the ECT electrodes. C) 48 hours after intervention contrast enhanced MRI reveals extended destruction of the complete metastasis respecting the tumor margins

volume can be partitioned to perform sequential treatments if the estimated duration of irradiation exceeds this limit [67]. After treatment is delivered, the brachytherapy catheters are removed.

Computed tomography-guided HDR IRT has generally a very low complication rate. Possible complications may be related to the insertion of the afterloading catheter, but using CT guidance should minimize this. Although IRT lessens radiation damage to healthy tissue due to precise targeting, radiation delivery and protection of radiosensitive organs such as the stomach or intestines are mandatory. To improve the protection of OARs, some groups reported early experience with the successful use of inflatable tissue separation devices [68]. Following IRT in very large tumors, the necrosis may cause temporary fever, chills, and nausea. These side effects may develop about 4-6 hours after the intervention and will disappear after a couple of hours under medication.

Interstitial interventional radiotherapy has some inherent advantages over both thermal ablation techniques and conventional radiotherapy. In contrast to thermal ablations, IRT has no limitations regarding the tumor size 
that can be treated. Several studies suggest that IRT is also suitable for the treatment of very large liver tumors. Further, the problem of heat dissipation does not occur with IRT. Therefore, high tumor blood flow and nearby larger blood vessels do not affect the success of IRT [67]. In addition, IRT is superior to conventional radiotherapy in terms of the precision of the local radiation delivery into cancer tissue while optimally sparing surrounding healthy tissue [69].

Interstitial interventional radiotherapy was reported to achieve good local tumor control in both primary liver tumors, e.g. hepatocellular carcinoma (HCC) and cholangiocellular carcinoma (CCC), and metastases [66].

In patients with HCC, IRT achieves excellent local tumor control (LTC) of up to $96.1 \%$ as well as a high survival benefit compared to the best supportive care (mOS 23 months vs. 5 months) [69]. Furthermore, in secondary liver malignancies, IRT obtains promising local tumor controls of 74.9-97.4\%, depending on the primary entity $(74.9-87.1 \%$ in colorectal cancer (CRC), $96.5-97.4 \%$ in breast cancer and $90 \%$ in pancreatic cancer liver metastases) $[69,70,71,72,73]$ (Figure 4$)$.
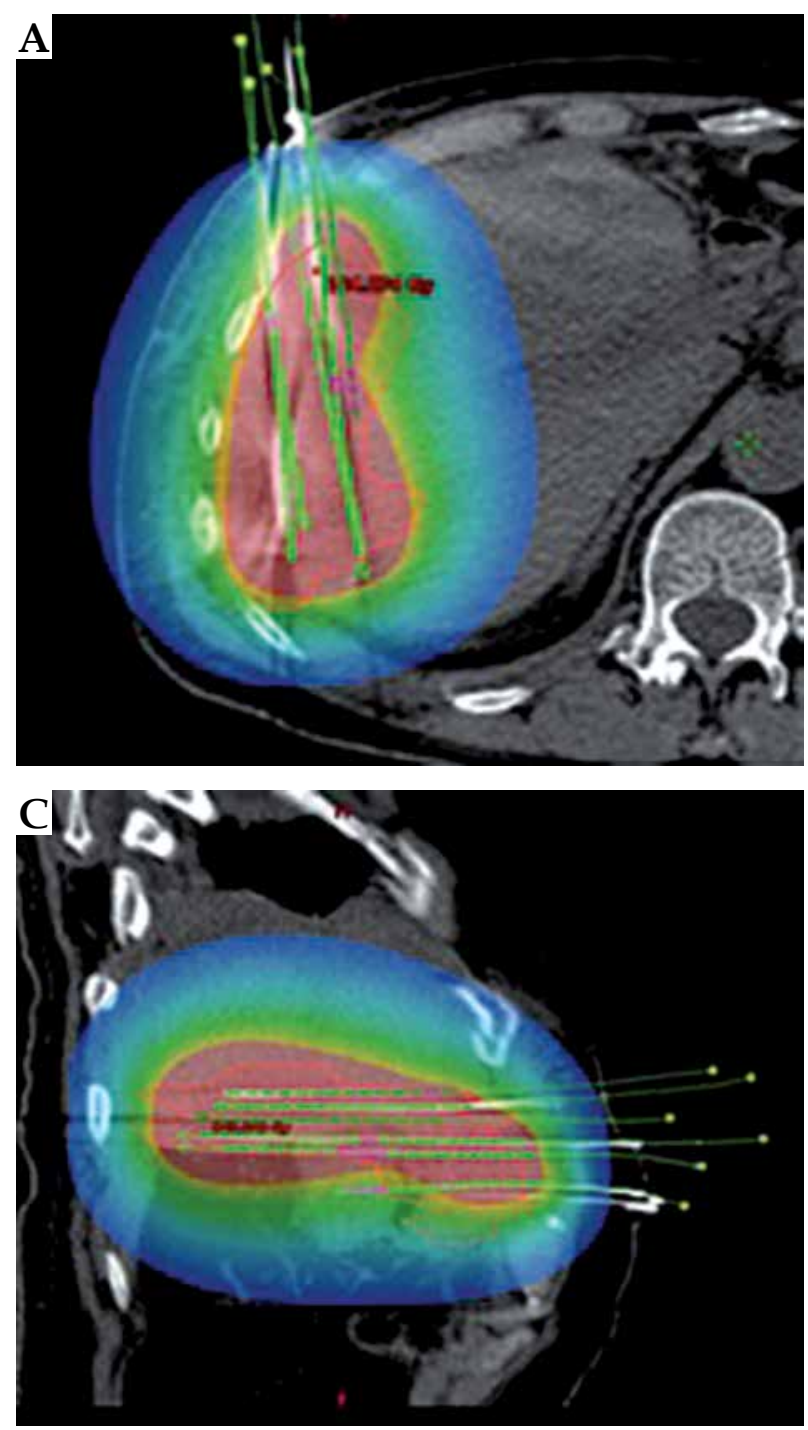

\section{Radioembolization}

Selective internal radiotherapy (SIRT) is the endovascular approach of delivering focal radiation that employs radioactive isotopes, e.g. yttrium-90 $\left({ }^{90} \mathrm{Y}\right)$-tagged glass or resin microspheres. SIRT is technically a whole-liver therapy. Tumor selectivity is achieved by the predominant supply of hypervascular malignancies by the hepatic artery [74]. After injection into the right or left branch of the hepatic artery, the microspheres become preferentially lodged in the arteriolar vasculature surrounding the tumor, delivering high doses of radiation to the surrounding area. Maximum tissue penetration for the pure beta-emitter ${ }^{90} \mathrm{Y}$ is $1.1 \mathrm{~cm}$. Absolute contraindications for SIRT are a potential for $\geq 30$ Gy radiation to be shunted to the lungs attested in the pretreatment technetium-99 $\left({ }^{99} \mathrm{Tc}\right)$ scan, inadvertent flow to the gastrointestinal tract that cannot be corrected with protective embolization, and prior radiation therapies of the liver. Unlike transarterial chemoembolization (TACE), main portal vein thrombus or obstruction is not a contraindication to radioembolization. Arterial ischemia after radioemboliza-

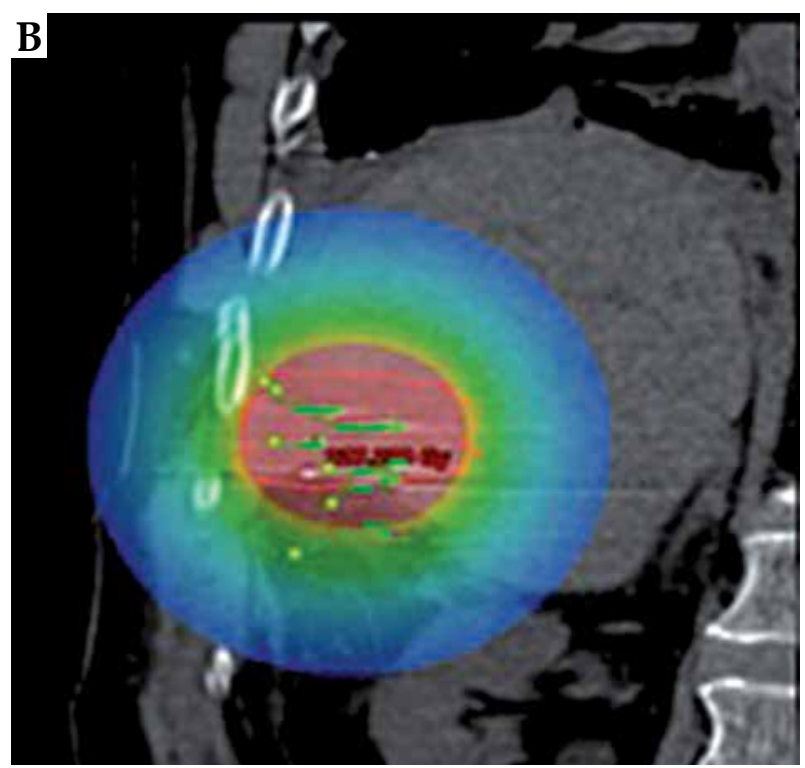

Fig. 4. Confluent colorectal liver metastases rapidly advancing under systemic chemotherapy and immunotherapy. Calculated radiation dose distribution after implantation of 11 brachytherapy applicators in transverse (A), coronal (B) and sagittal (C) planes 
tion based on the size of the beads is negligible, so the portal blood supply is less important from the standpoint of toxicity. In HCC SIRT induces extensive tumor necrosis with an acceptable safety profile [75]. However, there is no consensus as to the optimal use of this therapy, particularly when and if it should be chosen over TACE for treatment of unresectable HCC [76,77,78,79]. For patients who are eligible for TACE, generally TACE is still suggested. SIRT is preferred over TACE for patients with advanced HCC who are otherwise adequate candidates for local embolization therapy but who have macrovascular invasion, such as portal vein thrombus [80]. In symptomatic patients with metastatic carcinoid and pancreatic NET SIRT is also an alternative palliative technique to medical therapy alone [81,82]. In metastatic CRC randomized trials suggest that SIRT combined with systemic therapy does not improve survival as compared with chemotherapy alone and is associated with more adverse events. In the combined analysis of all three trials (SIRFLOX, FOXFIRE, and FOXFIRE-Global, 1103 patients, 549 receiving chemotherapy alone and 554 receiving chemotherapy plus SIRT), at a median follow-up of 43 months, despite having a higher objective response rate with combined therapy ( $72 \%$ vs. $63 \%)$, this did not translate into improved median overall survival (22.6 vs. 23.3 months, hazard ratio [HR] 1.04, 95\% CI: 0.9-1.19) or progression-free survival (11 vs. 10.3 months, HR 0.90, 95\% CI: 0.79-1.02), or a greater likelihood of subsequent liver resection. Furthermore, combined treatment was associated with significantly more grade 3 or 4 adverse events (especially hematologic toxicity). Of the 11 treatment-related deaths on study, eight were in the chemotherapy plus SIRT group, three of which were attributed to radiation-induced liver disease $[83,84,85,86]$. Further studies have to define the role of SIRT as consolidation therapy after chemotherapy.

\section{Economic aspects}

The cost evaluation of different treatment methods is very complicated and the result depends on many vari-

Table 1. Reimbursement of different non-surgical percutaneous tissue ablation methods in Germany (2019)

\begin{tabular}{lcc}
$\begin{array}{l}\text { Liver treatment } \\
\text { (transdermal/ } \\
\text { percutaneous) }\end{array}$ & $\begin{array}{c}\text { Reimbursement* in Germany } \\
\text { (2 nights in hospital stay) }\end{array}$ & DRG \\
\hline RFA & $€ 4046.33$ & H06C \\
\hline MWA & $€ 4046.33$ & H06C \\
\hline LITT & $€ 3434.43$ & H41D \\
\hline CRYO & $€ 3434.43$ & H41D \\
\hline IRE & $€ 7675.29$ & H09B \\
\hline ECT & $€ 3434.43$ & H41D \\
\hline HDR IRT & $€ 3331.85$ & H16B
\end{tabular}

RFA - radiofrequency ablation, MWA - microwave ablation, LITT - laser interstitial thermal therapy, CRYO - cryoablation, IRE - electroporation, ECT - electrochemotherapy, HDR IRT - high-dose-rate interventional radiotherapy ables [87]. Halpern et al. performed a comparative cost analysis in the USA [88], which resulted in median cost with SBRT in $\$ 27,145$ compared to $\$ 17,183$ for brachytherapy, $\$ 37,090$ for IMRT and $\$ 54,706$ for proton beam therapy $(p<0.001)$.

We also present the actual reimbursement costs for the different local ablation methods of percutaneous liver tumor ablations in Germany (Table 1).

\section{Status of education in interventional oncology}

Patients with cancer deserve evidence-based care, including the delivery of high-quality, high-value local treatments. Continued education is mandatory to advance awareness of and expertise in the full spectrum of interventional oncology applications $[89,90]$. In the interventional radiology community the need of education and quality assurance was realized [91,92] and CIRSE (Cardiovascular and Interventional Radiology Society of Europe) published recommendations for IR. Although basic knowledge of stereotactic and interventional radiotherapy is included in the published "European Curriculum and Syllabus for Interventional Oncology", no detailed knowledge of this method is disseminated in the organ-specific chapters [93].

In radiotherapy, ESTRO (European Society for Radiotherapy and Oncology) and GEC (Groupe Européen de Curiethérapie) offer multidisciplinary teaching courses and organ-specific treatment recommendations, although IO specific activities are rare. However, at the national society level, there is a growing number of activities to improve the situation $[94,95,96,97]$.

\section{Discussion}

Thermal ablation techniques are emerging as alternative treatment options to open surgery, being a well-recognized treatment option for both primary and secondary hepatic tumors [22]. However, hyperthermia-based technologies have some limitations, including heat sink effects in the vicinity of large blood vessels, the risk of causing cholestasis when treating lesions close to the thermosensitive bile ducts or damaging critical structures if proximal to the hepatic portal Glisson's capsule or diaphragm, or if located on the intra-abdominal free surface [98]. Since the liver is an organ of particular interest in interventional oncology, non-thermal ablation (NTA) techniques have the potential to complement local tumor therapy beyond the limitations of already established thermal ablations such as RFA or MWA. The lack of thermonecrosis and the preservation of collagenous structures enables NTA to be used near sensitive structures such as vessels, and bile ducts and may open up new opportunities for minimally invasive treatments in selected settings, e.g. large liver tumors located at the hilum of the liver.

Electrochemotherapy and IRT both have resemblances with endovascular interventional oncologic therapies. Similar to TACE, ECT enables controlled locoregional chemotherapy without marked systemic side effects. Using MEP, ECT has the potential to achieve more ho- 
mogeneous concentrations of a cytostatic drug in big lesions, assuming that response rates achieved with ECT may exceed those achieved with TACE [99]. Analogous IRT resembles selective internal radiation therapy (SIRT), allowing a welldefined focal to regional high-dose radiation preserving the functional reserve of the healthy liver remnant. In other anatomic sites, IRT was also proven as a successful treatment in the elderly and frail population $[100,101]$.

This potential advantage of HDR-IRT seems to be more important if we have a look at the recent publication of Gomez et al. reporting local treatments in patients with oligometastatic non-small cell lung cancer that did not progress after front-line systemic therapy, with prolonged progressionfree survival and overall survival obtained compared to maintenance therapy or observation. Even if the randomized phase II study of Palma et al. showed a significant increase in terms of overall survival for oligometastatic disease with stereotactic ablative radiotherapy (SABR), the treatment was associated with death related to the procedure in $4.5 \%$ of patients $(3 / 66)$. Toxicities causing deaths were related to radiotherapy (radiation pneumonitis, pulmonary abscess and subdural hemorrhage after surgery to repair a SABR-related perforated gastric ulcer) [14]. Comparable toxicities are not reported in the HDR IRT literature.

\section{Conclusions}

Despite the growing evidence for practicing hypofractionated regimes in many solid malignancies as well as the inherent benefit of delivering a high dose with a rapid dose fall-of, the limited use of IRT in comparison to conformal (3D-EBRT) or stereotactic external beam (SBRT) radiotherapy may be due to its invasive approach, operative risk, technical challenge, and long learning curve. Multicenter databases are mandatory for providing scientific evidence for eventually increasing the use of IRT in clinical practice, including for liver lesions.

Because of the need of the experience of different specialties, successful IRT is usually performed in a multidisciplinary cooperation by interventional radiologists and interventional radiation therapy experts. In such multidisciplinary groups, the potential of advising/applying the best fitting personalized local ablation method is also mandatory.

\section{Disclosure}

The authors report no conflict of interest.

\section{References}

1. Mattiucci GC, Autorino R, Tringali A et al. A phase I study of high-dose-rate intraluminal brachytherapy as palliative treatment in extrahepatic biliary tract cancer. Brachytherapy 2015; 14: 401-404.

2. Autorino, R, Mattiucci GC, Ardito F et al. Radiochemotherapy with gemcitabine in unresectable extrahepatic cholangiocarcinoma: long-term results of a phase ii study. Anticancer Res 2016; 36: 737-740.

3. Macchia G, Deodato F, Cilla $S$ et al. Volumetric intensity modulated arc therapy for stereotactic body radiosurgery in oligometastatic breast and gynecological cancers: Feasibility and clinical results. Oncol Rep 2014; 32: 2237-2243.

4. Rhim H, Goldberg SN, Dodd GD et al. Essential techniques for successful radio-frequency thermal ablation of malignant hepatic tumors. Radiographics 2001; 21: S17-35, discussion: S36-39.

5. Tombesi P, Di Vece F, Bianchi L, Sartori S. Thermal ablation of liver tumors: How the scenario was changed in the last decade. EMJ Hepatol 2018; 6: 88-94.

6. Damm R, Zörkler IM, Rogits B et al. Needle track seeding in hepatocellular carcinoma after local ablation by high-doserate brachytherapy: a retrospective study of 588 catheter placements. J Contemp Brachytherapy 2018; 10: 516-521.

7. Feng K, Ma KS. Value of radiofrequency ablation in the treatment of hepatocellular cancer. World J Gastroenterol 2014; 20: 5987-5998.

8. Tanaka M, Ando E, Simose S et al. Radiofrequency ablation combined with transarterial chemoembolization for intermediate hepatocellular carcinoma. Hepatol Res 2014; 44: 194200.

9. Mohnike K, Steffen IG, Seidensticker M et al. Radioablation by image-guided (HDR) brachytherapy and transarterial chemoembolization in hepatocellular carcinoma: a randomized phase II trial. Cardiovasc Intervent Radiol 2019; 42: 239249.

10. Kovács G, Tagliaferri L, Lancellotta V et al. Interventional oncology: should interventional radiotherapy (brachytherapy) be integrated into modern treatment procedures? Turk J Oncol 2019; 34: 16-22.

11. Schnapauf D, Collettini F, Steffen I et al. Activity-based cost analysis of hepatic tumor ablation using CT-guided highdose rate brachytherapy or CT-guided radiofrequency ablation in hepatocellular carcinoma. Radiat Oncol 2016; 11: 26.

12. Dawood O, Mahadevan A, Godman KA. Stereotactic body radiation therapy for liver metastases. Eur J Cancer 2009; 45: 2947-2959.

13. Gomez DR, Tang C, Zhang J et al. Local consolidative therapy vs. maintenance therapy or observation for patients with oligometastatic non-small-cell lung cancer: long-term results of a multi-institutional, phase II, randomized study. J Clin Oncol 2019; 37: 1558-1565.

14. Palma DA, Olson R, Harrow $S$ et al. Stereotactic ablative radiotherapy versus standard of care palliative treatment in patients with oligometastatic cancers (SABR-COMET): a randomised, phase 2, open-label trial. Lancet 2019; 393: 2051-2058.

15. Ricke J, Wust P, Wieners G et al. Liver malignancies: CT-guided interstitial brachytherapy in patients with unfavorable lesions for thermal ablation. I Vasc Interv Radiol 2004; 15: 1279-1286.

16. Ohri N, Tomé WA, Méndez Romero A et al. Local control after stereotactic body radiation therapy for liver tumors. Int J Radiat Oncol Biol Phys 2018; pii: S0360-3016(17)34525-X.

17. Hulstaert F, Mertens AS, Obyn $C$ et al. KCE REPORT 198CS: Innovative Radiotherapy Techniques: a multicentre time-driven activity-based costing study. 2013. https:/ / kce.docressources.info/index.php?lvl=notice_display\&id=3169 (accessed: 06.07.2019).

18. Carr BI, Kondragunta V, Buch SC et al. Therapeutic equivalence in survival for hepatic arterial chemoembolization and yttrium 90 microsphere treatments in unresectable hepatocellular carcinoma: a two-cohort study. Cancer 2010; 116: 1305-1314.

19. Rühl R, Lüdemann L, Czarnecka A et al. Radiobiological restrictions and tolerance doses of repeated single-fraction HDR-irradiation of intersecting small liver volumes for recurrent hepatic metastases. Radiat Oncol 2010; 5: 44. 
20. Crocetti L, Iezzi R, Goldberg SN et al. The ten commandments of liver ablation: expert discussion and report from Mediterranean Interventional Oncology (MIOLive) congress 2017. Eur Rev Med Pharmacol Sci 2018; 22: 3896-3904.

21. Brace CL. Radiofrequency and microwave ablation of the liver, lung, kidney, and bone: what are the differences? Curr Probl Diagn Radiol 2009; 38: 135-143.

22. Goldberg SN, Gazelle GS, Mueller PR. Thermal ablation therapy for focal malignancy: a unified approach to underlying principles, techniques, and diagnostic imaging guidance. AJR Am J Roentgenol 2000; 174: 323-331.

23. Poulou LS, Botsa E, Thanou I et al. Percutaneous microwave ablation vs radiofrequency ablation in the treatment of hepatocellular carcinoma. World J Hepatol 2015; 7: 1054-1063.

24. Crocetti L, De Baere T, Lencioni R. Quality improvement guidelines for radiofrequency ablation of liver tumours. Cardiovasc Intervent Radiol 2010; 33: 11-17.

25. Livraghi T, Meloni F, Di Stasi M et al. Sustained complete response and complications rates after radiofrequency ablation of very early hepatocellular carcinoma in cirrhosis: Is resection still the treatment of choice? Hepatology 2008; 47: 82-89.

26. Brunello F, Veltri A, Carucci P et al. Radiofrequency ablation versus ethanol injection for early hepatocellular carcinoma: a randomized controlled trial. Scand J Gastroenterol 2008; 43: 727-735

27. Zhou Y, Zhao Y, Li B et al. Meta-analysis of radiofrequency ablation versus hepatic resection for small hepatocellular carcinoma. BMC Gastroenterol 2010; 10: 78.

28. Imai K, Beppu T, Chikamoto A et al. Comparison between hepatic resection and radiofrequency ablation as first-line treatment for solitary small-sized hepatocellular carcinoma of $3 \mathrm{~cm}$ or less. Hepatol Res 2013; 43: 853-864.

29. Choi D, Lim HK, Rhim $\mathrm{H}$ et al. Percutaneous radiofrequency ablation for early-stage hepatocellular carcinoma as a first-line treatment: long-term results and prognostic factors in a large single-institution series. Eur Radiol 2007; 17: 684-692.

30. De Baere T, Risse $\mathrm{O}$, Kuoch V et al. Adverse events during radiofrequency treatment of 582 hepatic tumors. AJR Am J Roentgenol 2003; 181: 695-700.

31. Livraghi T, Solbiati L, Meloni F et al. Percutaneous radiofrequency ablation of liver metastases in potential candidates for resection: the 'test-of-time approach'. Cancer 2003; 97: 3027-3035.

32. Shibata T, Iimuro $Y$, Yamamoto $Y$ et al. Small hepatocellular carcinoma: comparison of radio-frequency ablation and percutaneous microwave coagulation therapy. Radiology 2002; 223: 331-337.

33. Shibata T, Niinobu T, Ogata N, Takami M. Microwave coagulation therapy for multiple hepatic metastases from colorectal carcinoma. Cancer 2000; 89: 276-284.

34. Ohmoto K, Yoshioka N, Tomiyama Y et al. Comparison of therapeutic effects between radiofrequency ablation and percutaneous microwave coagulation therapy for small hepatocellular carcinomas. J Gastroenterol Hepatol 2009; 24: 223-227.

35. Zhang L, Wang N, Shen Q et al. Therapeutic efficacy of percutaneous radiofrequency ablation versus microwave ablation for hepatocellular carcinoma. PLoS One 2013; 8: e76119.

36. Qian GJ, Wang N, Shen Q et al. Efficacy of microwave versus radiofrequency ablation for treatment of small hepatocellular carcinoma: experimental and clinical studies. Eur Radiol 2012; 22: 1983-1990.

37. Huo YR, Eslick GD. Microwave ablation compared to radiofrequency ablation for hepatic lesions: a meta-analysis. J Vasc Interv Radiol 2015; 26: 1139-1146.
38. Iezzi R, Pompili M, Posa A et al. Combined locoregional treatment of patients with hepatocellular carcinoma: State of the art. World J Gastroenterol 2016; 22: 1935-1942.

39. Iezzi R, Pompili M, La Torre MF et al. Radiofrequency ablation plus drug-eluting beads transcatheter arterial chemoembolization for the treatment of single large hepatocellular carcinoma. Dig Liver Dis 2015; 47: 242-248.

40. Iezzi R, Pompili M, Posa A et al. Interventional oncology treatments for unresectable early stage HCC in patients with a high risk for intraprocedural bleeding: Is a single-step combined therapy safe and feasible? Eur J Radiol 2019; 114: 32-37.

41. Weber SM, Lee FT Jr, Chinn DO et al. Perivascular and intralesional tissue necrosis after hepatic cryoablation: results in a porcine model. Surgery 1997; 122: 742-747.

42. Kerkar S, Carlin AM, Sohn RL et al. Long-term follow up and prognostic factors for cryotherapy of malignant liver tumors. Surgery 2004; 136: 770-779.

43. Lucatelli P, Iezzi R, De Rubeis G et al. Immuno-oncology and interventional oncology: a winning combination. The latest scientific evidence. Eur Rev Med Pharmacol Sci 2019; 23: 5343-5350.

44. Sarantou T, Bilchik A, Ramming KP. Complications of hepatic cryosurgery. Semin Surg Oncol 1998; 14: 156-162.

45. Ravikumar TS, Kane R, Cady B et al. Hepatic cryosurgery with intraoperative ultrasound monitoring for metastatic colon carcinoma. Arch Surg 1987; 122: 403-409.

46. Wang $\mathrm{C}$, Wang $\mathrm{H}$, Yang $\mathrm{W}$ et al. Multicenter randomized controlled trial of percutaneous cryoablation versus radiofrequency ablation in hepatocellular carcinoma. Hepatology 2015; 61: 1579-1590.

47. Thacker PG, Callstrom MR, Curry TB et al. Palliation of painful metastatic disease involving bone with imaging-guided treatment: comparison of patients' immediate response to radiofrequency ablation and cryoablation. $A J R$ Am J Roentgenol 2001; 197: 510-515.

48. Fairchild AH, Tatli S, Dunne RM et al. Percutaneous cryoablation of hepatic tumors adjacent to the gallbladder: assessment of safety and effectiveness. I Vasc Interv Radiol 2014; 25: 1449-1455.

49. Hinshaw JL, Lubner MG, Ziemlewicz TJ et al. Percutaneous tumor ablation tools: microwave, radiofrequency, or cryoablation - what should you use and why? Radiographics 2014; 34: 1344-1362.

50. Glazer DI, Tatli S, Shyn PB et al. Percutaneous image-guided cryoablation of hepatic tumors: single-center experience with intermediate to long-term outcomes. AJR Am J Roentgenol 2017; 209: 1381-1389.

51. Pacella CM, Bizzarri G, Magnolfi F et al. Laser thermal ablation in the treatment of small hepatocellular carcinoma: results in 74 patients. Radiology 2001; 221: 712-720.

52. Di Costanzo GG, D'Adamo G, Tortora R et al. A novel needle guide system to perform percutaneous laser ablation of liver tumors using the multifiber technique. Acta Radiol 2013; 54: 876-881.

53. Tombesi P, Di Vece F, Sartori S. Laser ablation for hepatic metastases from neuroendocrine tumors. AJR Am J Roentgenol 2015; 204: W732.

54. Orlacchio A, Bolacchi F, Chegai F et al. Comparative evaluation of percutaneous laser and radiofrequency ablation in patients with HCC smaller than $4 \mathrm{~cm}$. Radiol Med 2014; 119: 298-308.

55. Lencioni R, Cioni D, Della Pina C, Crocetti L. Hepatocellular carcinoma: new options for image-guided ablation. J Hepatobiliary Pancreat Sci 2010; 17: 399-403.

56. Cannon R, Ellis S, Hayes D et al. Safety and early efficacy of irreversible electroporation for hepatic tumors in proximity to vital structures. J Surg Oncol 2013; 107: 544-549. 
57. Lencioni R, Crocetti L, Narayanan G. Irreversible electroporation in the treatment of hepatocellular carcinoma. Tech Vasc Interv Radiol 2015; 18: 135-139.

58. Probst U, Fuhrmann I, Beyer L et al. Electrochemotherapy as a New Modality in Interventional Oncology: a review. Technol Cancer Res Treat 2018; 1: 1-12.

59. Jaroszeski M, Dang V, Pottinger $C$ et al. Toxicity of anticancer agents mediated by electroporation in vitro. Anticancer Drugs 2000; 11: 201-208.

60. Marty M, Sersa G, Garbay J et al. Electrochemotherapy - an easy, highly effective and safe treatment of cutaneous and sub- cutaneous metastases: results of ESOPE (European Standard Operating Procedures of Electrochemotherapy) study. EJC Suppl 2006; 4: 3-13.

61. Edhemovic I, Gadzijev E, Brecelj E et al. Electrochemotherapy: a new technological approach in treatment of metastases in the liver. Technol Cancer Res Treat 2011; 10: 475-485.

62. Djokic M, Cemazar M, Popovic P et al. Electrochemotherapy as treatment option for hepatocellular carcinoma, a prospective pilot study. Eur J Surg Oncol 2018; 44: 651-657.

63. Edhemovic I, Brecelj E, Gasljevic G et al. Intraoperative electrochemotherapy of colorectal liver metastases. J Surg Oncol 2014; 110: 320-327.

64. Gasljevic G, Edhemovic I, Cemazar M et al. Histopathological findings in colorectal liver metastases after electrochemotherapy. PLos One 2017; 12: e0180709.

65. Kowalik L, Lyczek J, Sawicki M. Individual applicator for brachytherapy for various sites of superficial malignant lesions. J Contemp Brachytherapy 2013; 5: 45-49.

66. Collettini F, Gebauer B. CT-guided high dose rate brachytherapy ablation of liver metastases. Imaging Med 2013; 5: 383-388.

67. Bretschneider T, Ricke J, Gebauer B, Streitparth F. Imageguided high dose rate brachytherapy of malignances in various organstechnique, indications and perspectives. J Contemp Brachytherapy 2016; 8: 251261.

68. Hass P, Steffen IG, Powerski M et al. First report on extended distance between tumor lesion and adjacent organs at risk using interventionally applied balloon catheters: a simple procedure to optimize clinical target volume covering effective isodose in interstitial high-dose-rate brachytherapy of liver malignomas. J Contemp Brachytherapy 2019; 11: 152-161.

69. Skowronek J. Current status of brachytherapy in cancer treatment - short review. J Contemp Brachytherapy 2017; 9 581-589.

70. Collettini F, Schnapauff D, Poellinger A et al. Hepatocellular carcinoma: computed-tomography-guided high-dose-rate brachytherapy (CT-HDRBT) ablation of large $(5-7 \mathrm{~cm})$ and very large (> 7 cm) tumours. Eur Radiol 2012; 22: 1101-1109.

71. Collettini F, Lutter A, Schnapauff D et al. Unresectable colorectal liver metastases: percutaneous ablation using CT-guided high-dose-rate brachytherapy (CT-HDBRT). Rofo 2014; 186: 606-612.

72. Ricke J, Mohnike K, Pech M et al. Local response and impact on survival after local ablation of liver metastases from colorectal carcinoma by computed tomography-guided highdose-rate brachytherapy. Int J Radiat Oncol Biol Phys 2010; 78: 479-485.

73. Wieners G, Mohnike K, Peters N et al. Treatment of hepatic metastases of breast cancer with CT-guided interstitial brachytherapy - a phase II-study. Radiother Oncol 2011; 100: 314-339.

74. Wieners G, Schippers A, Collettini F et al. CT-guided highdose-rate brachytherapy in the interdisciplinary treatment of patients with liver metastases of pancreatic cancer. Hepatobiliary Pancreat Dis Int 2015; 14: 530-538.
75. Vente MA, Wondergem M, van der Tweel I et al. Yttrium-90 microsphere radioembolization for the treatment of liver malignancies: a structured meta-analysis. Eur Radiol 2009; 19: 951-959.

76. EASL Clinical Practice Guidelines: Management of hepatocellular carcinoma. J Hepatol 2018; 69: 182-236.

77. Salem R, Gabr A, Riaz A et al. Institutional decision to adopt Y90 as primary treatment for hepatocellular carcinoma informed by a 1,000-patient 15-year experience. Hepatology 2018; 68: 1429-1440.

78. Bilbao JL, Iezzi R, Goldberg SN et al. The ten commandments of hepatic radioembolization: expert discussion and report from Mediterranean Interventional Oncology (MIOLive) congress 2017. Eur Rev Med Pharmacol Sci 2017; 21: 4014-4021.

79. Abdel-Rahman OM, Elsayed Z. Yttrium-90 microsphere radioembolisation for unresectable hepatocellular carcinoma. Cochrane Database Syst Rev 2016; 2: CD011313.

80. Gil-Alzugaray B, Chopitea A, Inarrairaegui M et al. Prognostic factors and prevention of radioembolization-induced liver disease. Hepatology 2013; 57: 1078-1087.

81. Kennedy A, Bester L, Salem R et al. Role of hepatic intra-arterial therapies in metastatic neuroendocrine tumours (NET): guidelines from the NET-Liver-Metastases Consensus Conference. HPB (Oxford) 2015; 17: 29-37.

82. Benson AB, 3rd, Geschwind JF, Mulcahy MF et al. Radioembolisation for liver metastases: results from a prospective 151 patient multi-institutional phase II study. Eur J Cancer 2013; 49: 3122-3130.

83. van Hazel GA, Heinemann V, Sharma NK et al. SIRFLOX: Randomized phase III trial comparing first-line mFOLFOX6 (plus or minus bevacizumab) versus mFOLFOX6 (plus or minus bevacizumab) plus selective internal radiation therapy in patients with metastatic colorectal cancer. J Clin Oncol 2016; 34: 1723-1731.

84. Dutton SJ, Kenealy N, Love SB et al. FOXFIRE protocol: an open-label, randomised, phase III trial of 5-fluorouracil, oxaliplatin and folinic acid (OxMdG) with or without interventional Selective Internal Radiation Therapy (SIRT) as first-line treatment for patients with unresectable liver-only or liver-dominant metastatic colorectal cancer. BMC Cancer 2014; 14: 497.

85. Gibbs P, Gebski V, Van Buskirk $M$ et al. Selective Internal Radiation Therapy (SIRT) with yttrium-90 resin microspheres plus standard systemic chemotherapy regimen of FOLFOX versus FOLFOX alone as first-line treatment of non-resectable liver metastases from colorectal cancer: the SIRFLOX study. BMC Cancer 2014; 14: 897.

86. Wasan HS, Gibbs P, Sharma NK et al. First-line selective internal radiotherapy plus chemotherapy versus chemotherapy alone in patients with liver metastases from colorectal cancer (FOXFIRE, SIRFLOX, and FOXFIRE-Global): a combined analysis of three multicentre, randomised, phase 3 trials. Lancet Oncol 2017; 18: 1159-1171.

87. Rahman F, Seung SJ, Cheng SY et al. Radiation costing methods: a systematic review. Curr Oncol 2016; 23: 392-408.

88. Halpern JA, Sedrakyan A, Hsu WC et al. Utilization, complications, and costs of stereotactic body radiation therapy (SBRT) for localized prostate cancer. Cancer 2016; 122: 2496-2504.

89. Petereit DG, Frank SJ, Viswanathan AN et al. Brachytherapy: where has it gone? J Clin Oncol 2015; 33: 980-982.

90. Kovács G, Tagliaferri L, Valentini V. Is an Interventional Oncology Center an advantage in the service of cancer patients or in the education? The Gemelli Hospital and INTERACTS experience. J Contemp Brachytherapy 2017; 9: 497-498.

91. Ghatan CE, Kuo WT, Hofmann LV, Kothary N. Making the case for early medical student education in interventional 
radiology: a survey of 2nd-year students in a single U.S. institution. J Vasc Interv Radiol 2010; 21: 549-553.

92. Tan ZB, Wang HJ, Zou R et al. Curriculum of interventional radiology for clinical medical undergraduates. Chin Med J 2017; 130: 2380-2381.

93. De Baère $\mathrm{T}$ (Editor in Chief). European Curriculum and Syllabus for Interventional Oncology. CIRSE Central Office, Vienna/Austria, 2019.

94. Tagliaferri L, Pagliara MM, Boldrini L et al. INTERACTS (INTErventional Radiotherapy ACtive Teaching School) guidelines for quality assurance in choroidal melanoma interventional radiotherapy (brachytherapy) procedures. J Contemp Brachytherapy 2017; 9: 287-295.

95. Tagliaferri L, Kovács G, Aristei $C$ et al. Current state of interventional radiotherapy (brachytherapy) education in Italy: Results of the INTERACTS survey. J Contemp Brachytherapy 2019; 11: 48-53.

96. Tagliaferri L, Budrukkar A, Lenkowicz J et al. ENT cobra ontology: the covariates classification system proposed by the Head \& Neck and Skin GEC-ESTRO Working Group for interdisciplinary standardized data collection in head and neck patient cohorts treated with interventional radiotherapy (brachytherapy). J Contemp Brachytherapy 2018; 10: 260-266.

97. Autorino R, Vicenzi L, Tagliaferri L et al. A national survey of AIRO (Italian Association of Radiation Oncology) brachytherapy (Interventional Radiotherapy) study group. J Contemp Brachytherapy 2018; 10: 254-259.

98. Curley S, Izzo F. Radiofrequency ablation of primary and metastatic hepatic malignancies. Int J Clin Oncol 2002; 7: 72-81.

99. Raoul JL, Forner A, Bolondi L et al. Updated use of TACE for hepatocellular carcinoma treatment: How and when to use it based on clinical evidence. Cancer Treat Rev 2019; 72: 28-36.

100. Lancellotta V, Kovács G, Tagliaferri L et al. Age is not a limiting factor in interventional radiotherapy (brachytherapy) for patients with localized cancer. Biomed Res Int 2018; 2178469.

101. Valentina L, Gyoergy K, Luca T et al. The role of personalized Interventional Radiotherapy (brachytherapy) in the management of older patients with non-melanoma skin cancer. J Geriatr Oncol 2019; 10: 514-517. 